\title{
Proline residues impart a tripartite segmental behavior to the nucleosomal H3 histone tail in the unmodified and posttranslationally modified states
}

\author{
Kathryn Piston \\ Syracuse University \\ Michael Cosgrove \\ SUNY Upstate Medical University \\ Shikha Nangia ( $\square$ snangia@syr.edu ) \\ Syracuse University https://orcid.org/0000-0003-1170-8461
}

\section{Article}

Keywords: histone tails, eukaryotic nucleosome, DNA

Posted Date: October 15th, 2021

DOI: https://doi.org/10.21203/rs.3.rs-964048/v1

License: (c) (i) This work is licensed under a Creative Commons Attribution 4.0 International License.

Read Full License 


\title{
Proline residues impart a tripartite segmental behavior to the nucleosomal H3 histone tail in the unmodified and posttranslationally modified states
}

\author{
Kathryn M. Piston ${ }^{\dagger}$, Michael S. Cosgrove ${ }^{\ddagger}$, and Shikha Nangia ${ }^{{ }^{\star}}$ \\ ${ }^{\dagger}$ Department of Biomedical and Chemical Engineering, Syracuse University, Syracuse NY 13244 \\ ${ }^{\ddagger}$ Department of Biochemistry and Molecular Biology, Upstate Medical University, Syracuse NY 13210
}

\begin{abstract}
Histone tails are integral structural and functional components of the eukaryotic nucleosome. These tails, rich in positively charged amino acid residues, interact with the DNA to stabilize the nucleosomal structure. However, capturing the biochemical effects of posttranslational modifications (PTMs) on histone tails in molecular detail using X-ray or NMR techniques remains a challenge due to their intrinsically disordered structure. In this work, we studied the $\mathrm{N}$-terminal portion of the $\mathrm{H} 3$ histone protein, a 38-residue tail, that when posttranslationally modified, is implicated in altering the tail's interaction with the DNA, affecting nucleosomal stability. Using all-atom molecular dynamics simulations for a total of 35 microseconds, we investigated the structure and dynamics of the wildtype $\mathrm{H} 3$ tail and seven known nucleosomal PTMs. Based on residues' contacts with DNA, water, and ions, dihedral angle analysis, and root-mean-square fluctuations of the tail residues, our results show that the $\mathrm{H} 3$ tail has a tripartite segmental nature. The three segments, labeled I, II, and III, are separated by the proline residues P16, P30, and P38. A comparison of wildtype H3 tail and proline-to-alanine-mutated $\mathrm{H} 3$ tail showed that the prolines function as segmental dividers or hinges of the H3 tail. We show that Segment I is more dynamic than Segments II and III, and Segment I makes multiple transient contacts with the DNA. The PTMs affect the tail's dynamics to different extents, but the tripartite segmental nature of the tail is preserved. Notably, single-residue modification of the lysine by acetylation or methylations in Segment I versus multiple residue modifications by serine phosphorylation or lysine methylations have marked effects on the tail's flexibility and interaction with the DNA. This study highlights the significance of proline residues in creating the segmental behavior of the $\mathrm{H} 3$ tail.
\end{abstract}

*Address for correspondence:

Professor Shikha Nangia

343 Link Hall

Department of Biomedical and Chemical Engineering

Syracuse University, Syracuse, NY 13244, USA

Phone (315) 4430571

Email: snangia@syr.edu

\section{ORCID}

Kathryn M. Piston: 0000-0001-5134-5509

Michael Cosgrove: 0000-0002-6818-6716

Shikha Nangia: 0000-0003-1170-8461 


\section{INTRODUCTION}

Histones are a family of proteins that package the DNA into chromatin in the nucleus of a eukaryotic cell. The basic unit of the chromatin is the nucleosome core particle (NCP) that contains two copies of four histones $(\mathrm{H} 2 \mathrm{~A}, \mathrm{H} 2 \mathrm{~B}, \mathrm{H} 3$, and $\mathrm{H} 4)$ in the form of an octameric core around which 146 DNA base pairs (or 1.7 turns) are wrapped. ${ }^{1-2}$ The NCP is stabilized by multiple electrostatic interactions between the negatively charged DNA and histones, abundant in positively charged lysine and arginine residues. ${ }^{3-6}$ The DNA wraps

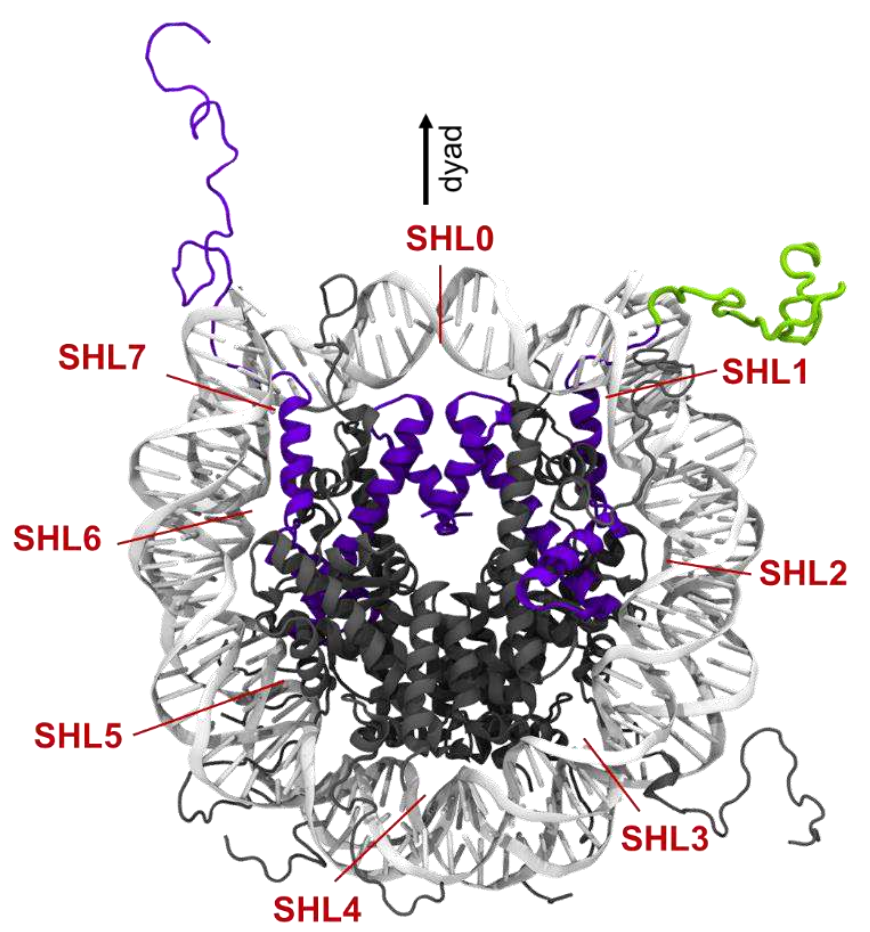

Figure 1. Atomistic structure of a nucleosome core (PDB:1KX5). Snapshot of view along the superhelix axis with the dyad axis point up (arrow). The 146 DNA base pair (light gray, ribbons) and core histone proteins. Color scheme: H2A-H2B (gray), H4 (gray), and H3.1 (purple). Superhelix locations (SHL) are indicated in red. The H3.1 tail at SHL 1 (green) is the focus of this work. around the histones to create unique histone-DNA binding sites called superhelical locations (SHL) where the DNA's minor grooves face the histone octamer (Figure 1). ${ }^{7-8}$

The nucleosomal histones have a welldefined secondary structure in the core with disordered tail region(s) that protrude from a unique position on the NCP. Each NCP has ten histone tails that include the $\mathrm{N}$-termini of the eight histones and two C-termini of H2A. The histone tails are solvent accessible, but the the available nuceosomal X-ray crystal structures or cryogenic electron microscopy structures are unable to resolve the tails. ${ }^{9-12}$ Several nuclear magnetic resonance (NMR) spectroscopy studies show that the $\mathrm{H} 2 \mathrm{~A}, \mathrm{H} 3$, and $\mathrm{H} 4$ tails remain dynamic in condensed nucleosomal arrays. ${ }^{13-15}$ It remains open to interpretation as to whether the tail is viewed as intrinsically disordered or adopts transient secondary structure motifs. ${ }^{16-19}$

The N-terminal $\mathrm{H} 3$ tails exit the nucleosomal core between the minor grove of the DNA gyres at SHL1 and SHL7 of the NCP. ${ }^{20}$ Sequence alignment of the $\mathrm{H} 3$ tail shows that both the length and the residue composition are conserved. ${ }^{21-22} \mathrm{An}$ unmodified wildtype $\mathrm{H} 3$ tail has 38 residues, and a net charge of +12 due to multiple arginine and lysine residues distributed over its length. Previous studies show that the $\mathrm{H} 3$ tails maintain fragile contacts with the nucleosomal DNA, even at increased salt concentrations. ${ }^{23-24}$ These DNA-tail contacts have been described as "fuzzy" because they cannot be described by a fixed conformation but rather present as an ensemble of multiple short-lived conformations ${ }^{25}$ However, posttranslational modifications (PTMs) such as acetylation, ${ }^{26}$ methylation, ${ }^{27}$ and phosphorylation ${ }^{28}$ of the tail residues change the physicochemical properties of the residues and their nonbonded interactions with the DNA. ${ }^{29-33}$ The PTMs also influence the internucleosomal interactions, chromatin organization, and nucleosomal stability. ${ }^{34-35}$ And compared to wildtype NCP, the NCPs with deleted $\mathrm{H} 3$ tails are unstable because the DNA is less tightly wrapped to the core histones and more accessible to enzymatic modifications. ${ }^{36-38}$

A recent review highlights $\mathrm{H} 3$ tail's structure, PTMs, and cell-cycle dynamics. ${ }^{39}$ Other key aspects of the $\mathrm{H} 3$ tail that have been reported are as follows. First, $\mathrm{H} 3$ tails lack secondary structure and collapse onto the NCP making robust yet transient contacts with the nucleosomal DNA. ${ }^{24,}{ }^{40-43}$ Second, NMR data 
suggests that $\mathrm{H} 3$ tails have a high degree of conformational flexibility and are highly mobile..$^{44-46}$ Third, modification(s) of the $\mathrm{H} 3$ tail in the form of PTMs or mutations can lead to altered tail dynamics. ${ }^{47-48}$ All three aspects of the $\mathrm{H} 3$ tail demonstrate that the high flexibility of the nucleosomal tails is an essential feature for their signaling and regulatory functions. ${ }^{49-50}$ Consequently, these aspects pose a challenge in characterizing their molecular features, interactions, and dynamics.

To address these challenges, computational molecular dynamics (MD) studies have focused on the peptides that match the $\mathrm{H} 3$ tail's residue sequence instead of the entire NCP. ${ }^{51-52}$ Such studies fail to provide a thorough understanding of the tail's characteristics due to the absence of the nucleosome. On the other hand, studies of modified H3 tail in the NCP also have been limited to only a few hundred nanoseconds due to the high computational cost of the sizable atomistic system. ${ }^{53}$ Thus, in this work, we investigated the single NCP using an extensive set of atomistic MD simulations for a total of $35 \mu \mathrm{s}$. The simulations included unmodified, modified, and mutated $\mathrm{H} 3$ tail. The modifications included common PTMs - serine phosphorylation, lysine methylation(s), and lysine acetylation. ${ }^{54}$ We used the CHARMM forcefield with explicit water, a decision informed by the availability of extensive research and discussion on force field selection. ${ }^{24,55}$ The relative binding strengths of the modified $\mathrm{H} 3$ tails were determined using the steered MD simulations. The analysis of the simulation data was performed using in-house codes.

Our results show that the N-terminal portion of the H3.1 (H3) tail at SHL1 adopts a collapsed conformation and is stabilized via multiple nucleosomal DNA contacts along the length of the tail. This behavior of the $\mathrm{H} 3$ tail is in excellent agreement with previously reported results based on experimental and computational techniques. ${ }^{44-50}$ Our significant conceptual advance in this work is the tripartite segmental behavior of the $\mathrm{H} 3$ tail caused by the hinge-like conformation rigidity of the proline residues at positions 16,30 , and 38 . This concept was further reinforced by investigating four additional systems where proline residues were mutated to alanine. The tail demonstrates segmental behavior, despite its collapsed conformation. Furthermore, although the PTMs can alter tail dynamics to different extents, the tripartite segmental behavior of the $\mathrm{H} 3$ tail is largely preserved.

\section{METHODS}

The $1.9 \AA$ resolution X-ray structure of human NCP (PDB: 1KX5) was used as the starting structure for all simulations. ${ }^{2}$ Five sets of simulations were performed: (1) an ensemble of short simulations to explore the interactions of unmodified (WT) H3 tails (SHL1 and SHL7) with DNA; (2) equilibrium simulation of the unmodified H3 tail; (3) H3 tail with proline to alanine mutations; (4) H3 tail with PTMs; and (5) steered MD simulations of the H3 tails. Details, such as simulation box size and box contents, and the simulation time for each system is provided in Table S1.

An ensemble of ten simulations was run for $30 \mathrm{~ns}$, using different random seeds following energy minimization to sample the initial conformational space and observe how the $\mathrm{H} 3$ tail interacts with the nucleosome. One WT H3 tail system was extended for $2.5 \mu \mathrm{s}$ for a detailed analysis of the equilibrated tail. The proline to alanine mutations systems were prepared in the SHL1 H3 tail before it collapsed on the DNA. The equilibrated WT NCP was then modified to generate seven different systems (Fi).

All simulations were performed using the GROMACS package ${ }^{56}$ and CHARMM36 forcefield ${ }^{57}$ as described in detail below. As with all simulations in this study, the set of ten systems was explicitly solvated with transferable intermolecular potential 3P (TIP3P) water ${ }^{58}$ and $150 \mathrm{mM} \mathrm{NaCl}$. The force field parameters for lysine acetylation and lysine mono, di, and trimethylations were obtained from the literature, ${ }^{59}$ and are reported in Figures S1-S4. The serine phosphorylation force field parameters were generated using the CHARMM GUI web server. ${ }^{60}$ All systems were explicitly solvated with TIP3P water in $150 \mathrm{mM} \mathrm{NaCl}$. Each system was energy minimized using the steepest descent algorithm, followed by $125 \mathrm{ps}$ of isothermalisochoric (NVT) at $310.15 \mathrm{~K}$, and $100 \mathrm{~ns}$ isothermal-isobaric (NPT) equilibration at $310.15 \mathrm{~K}$ and 1 bar pressure. A 2 fs integration time step was used. Following equilibration, the production runs were performed for $1 \mu \mathrm{s}$. The temperature was maintained at $310.15 \mathrm{~K}$ to mimic physiological body temperature using a Nose-Hoover thermostat ${ }^{61}$ with a 1.0 ps coupling constant. Isotropic pressure was maintained at 1 bar using 
the Parrinello-Rahman barostat ${ }^{62}$ with a 5.0 ps coupling constant and $4.5 \times 10^{-5}$ compressibility constant. The temperature was coupled separately for the protein, solvent and ions, and DNA groups. No position restraints were used in the production runs. The bonds with hydrogen atoms were constrained using the linear constraint solver (LINCS). Long-range electrostatic interactions were calculated using the particle mesh Ewald algorithm at $1.2 \mathrm{~nm}$ cutoff and van der Waal's interactions were calculated with a $1.2 \mathrm{~nm}$ cutoff.

Table 1. Details of the systems investigated in this work: the type of PTM, description of the PTM, length of simulation, and total number of atoms in the simulation box.

\begin{tabular}{llll}
\hline PTM $^{\mathbf{a}}$ & Description & $\boldsymbol{t}(\boldsymbol{\mu s})$ & \# Atoms \\
\hline $\mathrm{WT}$ & Wild type (Unmodified) & 2.5 & 308,041 \\
$\mathrm{~K}^{4} \mathrm{Ac}$ & Lysine 4 acetylation & 1 & 307,917 \\
$\mathrm{~K}^{4} \mathrm{Me1}$ & Lysine 4 monomethylation & 1 & 307,873 \\
$\mathrm{~K}^{4} \mathrm{Me2}$ & Lysine 4 dimethylation & 1 & 307,960 \\
$\mathrm{~K}^{4} \mathrm{Me3}$ & Lysine 4 trimethylation & 1 & 308,152 \\
$\mathrm{~K}^{9,27} \mathrm{Me3}$ & Lysine 9 \& 27 trimethylation & 1 & 307,893 \\
$\mathrm{~S}^{10,28} \mathrm{P}$ & Serine 10 \& 28 phosphorylation & 1 & 307,970 \\
$\mathrm{~K}^{4,36}{ }_{\mathrm{Me3}} \mathrm{K}^{9,27} \mathrm{Me1}$ & Lysine 4 \& 36 trimethylation + Lysine 9 \& 27 monomethylation & 1 & 308,038 \\
\hline
\end{tabular}

${ }^{a}$ monomethylation (Me1), dimethylation (Me2), trimethylation (Me3), acetylation (Ac), and phosphorylation (P)

Steered MD simulations were run for all systems referenced in Table S1. Each starting structure was created using the control system at $1.5 \mu \mathrm{s}$ into the trajectory. To reduce the computational burden, however, all histone proteins except for the $\mathrm{H} 3$ tail were deleted; the 147 base-pair DNA remained intact. The H3 tail and DNA structures were placed in a cuboidal box with dimensions $\left(10 \times 15 \times 30 \mathrm{~nm}^{3}\right)$ sufficient to provide space for the pulling simulations to take place along the z-axis. As with the explicit solvent simulations, TIP3P water in $150 \mathrm{mM} \mathrm{NaCl}$ water was used. Minimization and equilibration as described above was performed on each system prior to pulling. Following equilibration all restraints were removed except for those on the DNA which was used as the immovable reference for the pulling simulations. The histone $\mathrm{H} 3$ tail was pulled away from the DNA structure along the $z$-axis over $500 \mathrm{ps}$, using a spring constant of 1000 $\mathrm{kJ} \mathrm{mol}^{-1} \mathrm{~nm}^{-2}$ and a pull rate of $0.01 \mathrm{~nm} \mathrm{ps}^{-1}$ on the first 15 residues. A final center-of-mass (COM) distance between the peptides of approximately $15 \mathrm{~nm}$ was achieved. For this trajectory, snapshots spaced approximately $0.2 \mathrm{~nm}$ apart were used to generate the starting configurations for umbrella sampling windows. Since the COM between the $\mathrm{H} 3$ tail and the DNA began at $3 \mathrm{~nm}$, approximately 60 windows were used. In each window $50 \mathrm{~ns}$ of MD was performed for a total simulation time of $3 \mu$ s per system for umbrella sampling. Data were analyzed using the weighted histogram analysis method (WHAM).

The simulation trajectories and protein structures were analyzed using GROMACS utilities and in-house python scripts with the MDAnalysis package ${ }^{63-64}$ Root-mean-square fluctuation (RMSF) of $\mathrm{C}_{\alpha}$ atoms of the tail residues was computed for each system. All visual renderings were created using visual molecular dynamics version 1.9.3 (VMD) ${ }^{65}$ and PyMOL.

\section{RESULTS AND DISCUSSION}

\subsection{H3 tail at SHL1 adopts collapsed conformation}

We created an ensemble of ten independent WT systems to sample the conformational space of the two $\mathrm{H} 3$ tails of a single NCP. During the $30 \mathrm{~ns}$ simulations, the $\mathrm{H} 3$ tails located at SHL1 versus SHL7 behaved differently. In all systems the $\mathrm{H} 3$ tail located at SHL1 collapsed onto the nucleosomal DNA within the first $\sim 10 \mathrm{~ns}$ and remained collapsed for the rest of the simulation time (Figures 2a and S5). However, the SHL7 $\mathrm{H} 3$ tail that began extended often became tangled at the end of the DNA, indicating that linker DNA is required to study the SHL7 H3 tail appropriately. Thus, this work is focused only on features of the SHL1 
$\mathrm{H} 3$ tail in its modified, unmodified, and mutated forms; features of the SHL7 H3 tail will be studied in future work.

We found that the RMSF values of $C_{\alpha}$ atoms of SHL1 tail residues lie in the range 2-14 $\AA$, with the tip of the tail showing the highest fluctuations. The $\mathrm{H} 3$ tail in all ten systems showed a similar range of fluctuations, despite their randomly assigned initial velocities for MD run. Notably, the fluctuations of proline residues P16, P30, and P38 appeared to be smaller than the adjacent residues in all systems (Figure 2b). To investigate if the proline residues have a distinct role in the SHL1 H3 tail, we randomly selected one system in the ensemble and extended it to $2.5 \mu$ s for further analysis.

(a)
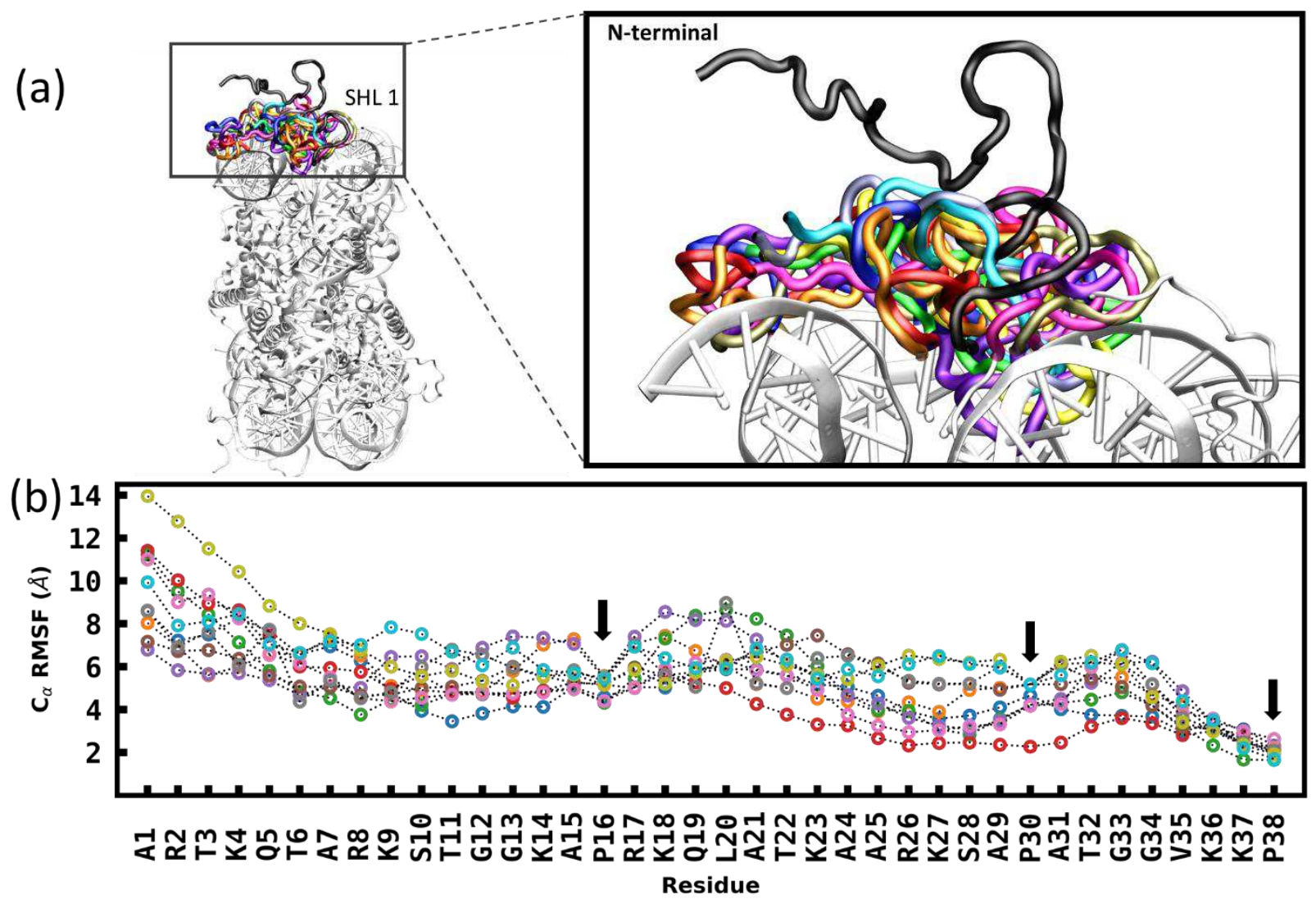

Figure 2. Conformational dynamics of SHL1 N-terminal H3 tail collapsed on the DNA. (a) Ten WT H3 tail conformations, shown in different colors after $30 \mathrm{~ns}$ of simulation. The reference tail conformation is shown in black, and the DNA is shown in light gray. (b) Root-mean-square fluctuation (RMSF) values of $\mathrm{C} \alpha$ atoms of the tail residues. The arrows indicate the low fluctuation values of the proline residues.

\subsection{Proline residues divide the $\mathrm{H} 3$ tail into three segments}

A notable feature of the $\mathrm{H} 3$ tail is the location of its three highly conserved proline residues-P16, $\mathrm{P} 30$, and P38 (Figure 3a). If we use the analogy of a human arm for the $\mathrm{H} 3$ tail, the locations of the proline residues map to wrist (P16), elbow (P30), and shoulder (P38) joints. And like arm joints, the prolines have restrictive dihedral angles that prevent the transition between their cis and trans conformations.

To confirm the restricted torsional angle in $\mathrm{H} 3$ tail's prolines, we generated Ramachandran plots for proline residues only in the WT and the seven PTMs system (Figure S6). In all systems, we observed that the phi $(\Phi)$ and psi $(\Psi)$ angles of the prolines have a narrow range, for $\Phi$ from about $-50^{\circ}$ to $-100^{\circ}$ and for $\Psi$ from $120^{\circ}$ to $160^{\circ}$, which match the trans conformational geometry around the proline residues. The torsional 
angle analysis confirms that the prolines function as hinges and maintain their trans conformation irrespective of the $\mathrm{H} 3$ tail's PTM state.

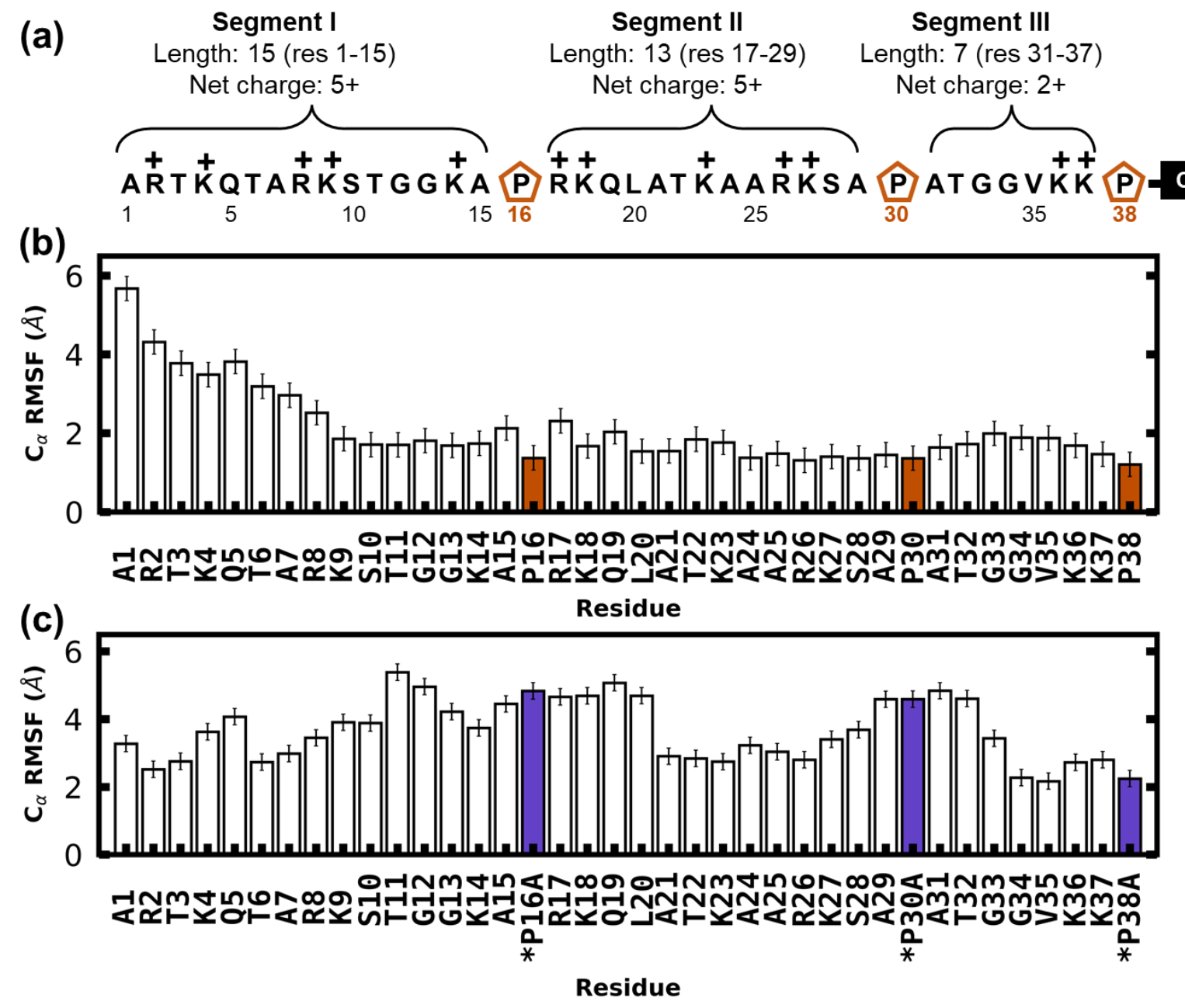

Figure 3. N-terminal portion of the SHL1 H3 tail. (a) Residue sequence and location of the charged residues in Segment I, II, and III. The prolines (orange) are segmental dividers. Bar plot of the root-mean-square fluctuation (RMSF) values of $\mathrm{C}_{\alpha}$ atoms of the tail residues in the (b) WT and (c) H3m (P16A, P30A, and P38A) systems computed over the last $500 \mathrm{~ns}$ of the simulation. The RMSF values of prolines are marked in the WT (orange) and $\mathrm{H} 3 \mathrm{~m}$ (purple) bar plots as a guide to the eye.

For further evidence that prolines are H3 tail's segmental dividers, we computed the RMSF values of the $\mathrm{C}_{\alpha}$ atoms of the tail residues in the WT system after $2.5 \mu \mathrm{s}$ of the simulation. Figure $3 \mathrm{~b}$ shows that the three prolines have RMSF values of $<2 \AA$, which are the lowest compared to all residues in the rest of the chain. Additional support for the $\mathrm{H} 3$ tail being segmental is indicated by the RMSF values of residues 1-15, which decrease progressively from 6 to $2 \AA$ with the RMSF lowest value at P16. This trend suggests that the proline may act as a hinge, and residues 1-15 (the free end of the H3 tail) fluctuate with little constraint. The midsection of the tail, residues 17-29, has an almost constant RMSF value of $2.2 \AA$, indicating constrained dynamics between P16 and P30. The third region between P30 and P38 also shows small RMSF values averaging $2.5 \AA$, indicating slightly higher dynamics than the midsection of the $\mathrm{H} 3$ tail while still being conformationally constrained.

To further investigate the conformational role of prolines, we created a system in which the $\mathrm{H} 3$ tail's proline residues were mutated $(\mathrm{H} 3 \mathrm{~m})$ to alanine $\mathrm{P} 16 \mathrm{~A}, \mathrm{P} 30 \mathrm{~A}$, and $\mathrm{P} 38 \mathrm{~A}$. The mutations were performed on the 
extended SHL1 H3 tail before it collapsed on the DNA. A comparison of the RMSF values of the H3m and WT systems shows strikingly different behavior of their tail residues. In H3m, the proline RMSF values are approximately a factor of two larger than in the WT system (Figure 3c). Other tail residues also have significantly larger RMSF values indicating greater tail dynamics if prolines are replaced with alanine residues. Notably, the segmental character observed in the WT tail was lost in H3m, indicating that the prolines act as hinges in the collapsed WT H3 tail. Additional support for proline's hinge-like behavior came from three systems in which the prolines were replaced one at a time: P16A only, P30A only, and P38A only. In all three cases, replacement of the proline by conformationally less retrictive alanine led to loss of local segmental behavior (Figures S7-S8).

Based on these results, we divided the H3 tail into three regions-Segment I (residues 1-15, 5+ charge), Segment II (residues 17-29; 5+ charge), and Segment III (residues 31-37; 2+ charge). Each segment is unequal in length, and charge or location of the positively charged residues (Figure 3a).

\subsection{H3 tail exhibits tripartite segmental behavior}

To examine the structural and functional differences among the $\mathrm{H} 3$ tail segments, we performed contact analyses of the residues with DNA, water, and counter ions. For all systems, the heatmaps of each residue's contacts with the DNA sugar-phosphate backbone (5 Å cutoff), surrounding water ( $3 \AA$ cutoff), sodium ions, and chloride ions were computed over the last $500 \mathrm{~ns}$. Several aspects of the H3 tail emerged from contact analysis that reinforced the $\mathrm{H} 3$ tail's segmental behavior.

First, the proline residues made very few contacts with the DNA, even P38 although it is closest to nucleosomal DNA when the $\mathrm{H} 3$ tail emerges from the NCP core. There was also minimal contact with water due to the hydrophobic nature of the proline. Moreover, the PTMs did not affect proline-DNA and water contacts, despite the proximity of S28 phosphorylation to P30 or K36 monomethylation to P38. Taken together, the contact heatmaps (Figures 4) and torsional analysis (Figure S9) reinforce our interpretation that the $\mathrm{H} 3$ tail's proline residues function as conformational hinges.

Second, the total number of DNA contacts progressively decreased along the length of the $\mathrm{H} 3$ tail from Segment I to Segment III. As evident from Figure 4a, Segment I, the free end of the H3 tail, makes several contacts, whereas Segment II, the midsection of the H3 tail, has three robust DNA contacts (A21, T22, and R26) and Segment III, the shortest section of $\mathrm{H} 3$ tail makes minimal contacts. The $\mathrm{H} 3$ tail is hydrated along its length, and like DNA contacts, water contacts are highest with Segment I residues and lowest with Segment III. The PTMs affect DNA and water contacts, but these changes occur locally in the proximity of a modified residue.

Furthermore, we analyzed the conformational flexibility of $\mathrm{H} 3$ tails by computing the end-to-end distance $\left(R_{e e}\right)$ and the radius of gyration $\left(R_{g}\right)$ of each segment (Figure S10). Among the three segments, the most significant variations were observed in Segment I while values remained almost unchanged for Segments II and III. Notably, in a previous study using NMR, the segmental behavior of the H3 tail was reported. ${ }^{26}$ 


\section{Segment I}

田

Segment II

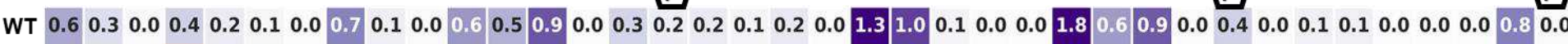

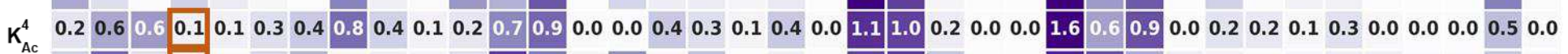

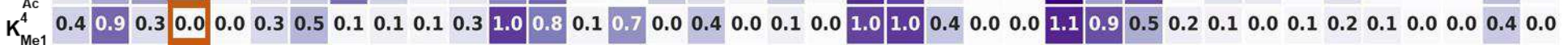

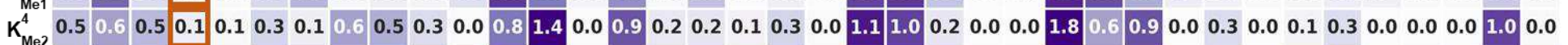

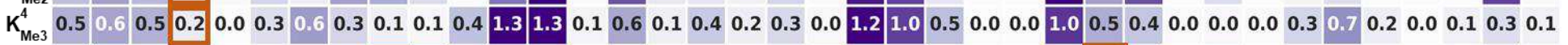

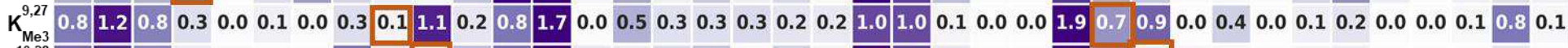

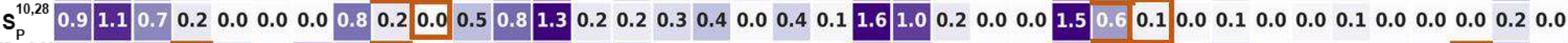

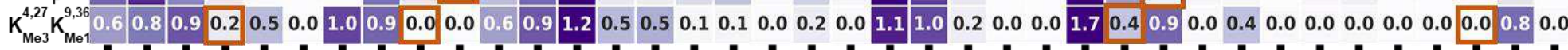

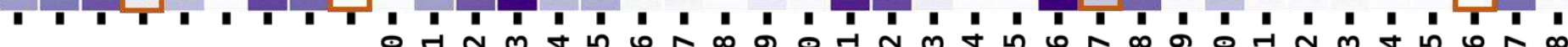

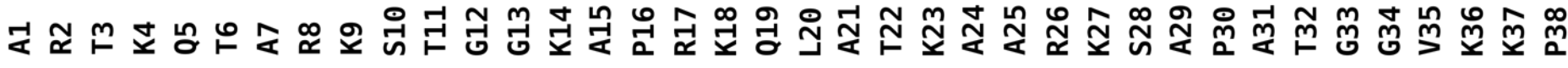

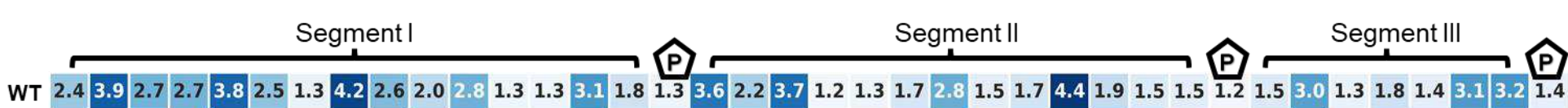

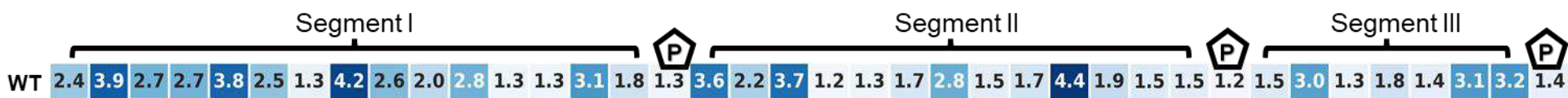

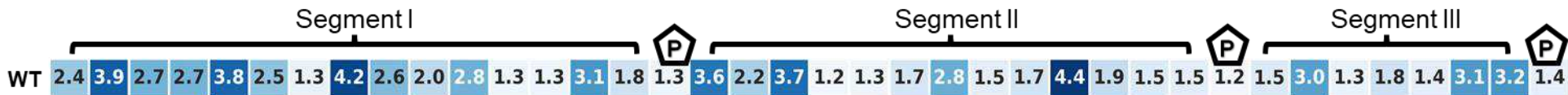

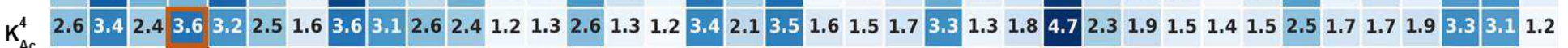

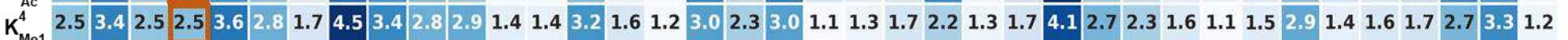

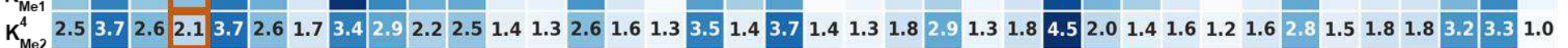

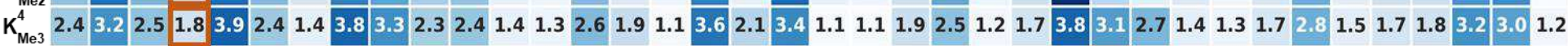

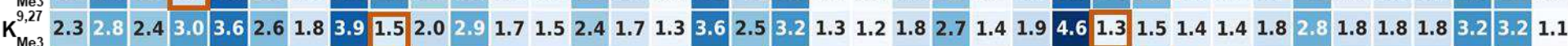

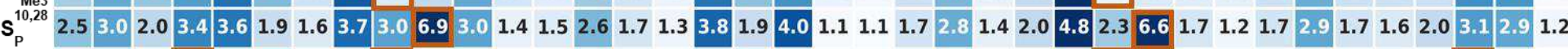

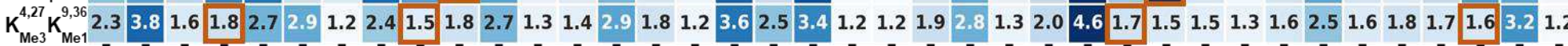

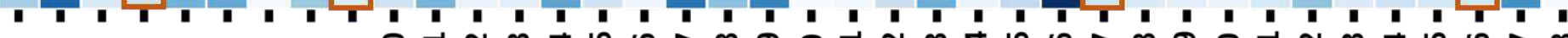

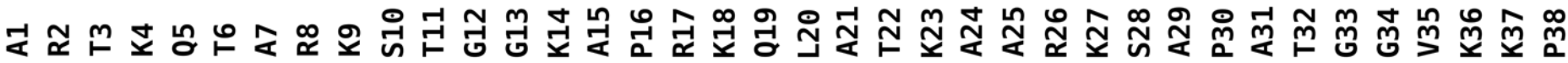

Figure 4. Contact map of $\mathrm{H} 3$ tail's residues with DNA (top) and water (bottom). The scales are shown on the right for both contact maps and represent the average number of contacts per residue over the last $500 \mathrm{~ns}$ of simulation. The modified residues in all PTM systems are marked with a red outline to guide the eye. 


\subsection{H3 tail's Segment I residues make fuzzy contacts with DNA}

Of the 15 residues in Segment I, the five charged (R2, K4, R8, K9, K14) and five polar (T3, Q5, T6, S10, T11) residues (approximately $67 \%$ of the segment) promote binding to the nucleosomal DNA. Also, the flexibility of the segment is enhanced because it is the free end of the H3 tail and lacks secondary structure. Consequently, DNA and water heatmaps show subtle differences in each residue's contact within Segment I, which were observed in all eight systems. The DNA-histone tail literature refers to such contacts as "fuzzy contacts" because the histone tails adopt an ensemble of thermally accessible conformations, causing the residue sidechains to establish multiple energetically favorable nonbonded interactions (for example, coulombic, h-bonding, salt bridges, and cation- $).{ }^{66}$ Interestingly, we observed fuzzy contacts only in Segment I. Although Segments II and III lack secondary structure, their conformations are restricted by the proline hinges.

To demonstrate that the fuzzy contacts can be short-lived (transient), long-lived (persistent), or noncontacting, we computed time-lapsed contacts of the R8 residue over $500 \mathrm{~ns}$ in 20 ns increments for all eight systems (Figure 5). We selected 20 ns increments to ensure we captured any fleeting interactions as well as the frequency with which a lost interaction can be reestablished within few nanoseconds, illustrating the fuzzy contacts. In the WT tail, the R8 residue is in contact with up to three DNA residues (d46, d47, and d68) at any instance of time and in almost constant contact with water. Contact of $\mathrm{R} 8$ with $\mathrm{Na}^{+}$and $\mathrm{Cl}^{-}$is rare and instantaneous, lasting not more than 20 ns.

Fuzzy contacts are also apparent in the PTM systems, although each system has different contacts. Even a single sidechain modification, whether it is acetylation or mono-, di-, or tri-methylation drastically impacts the R8's contacts. For example, the K4 acetylation causes R8 to make transient contacts with approximately six DNA residues, none of which last more than 200 ns (Figure 5). Compared to WT, acetylation causes a slight weakening of R8 contact with DNA, while contacts with water and ions are unchanged.

In contrast, $\mathrm{K} 4$ monomethylation eliminates R8-DNA and R8- $\mathrm{Na}^{+}$contacts. It appears that monomethylation at K4 detaches R8 from the DNA and causes it to become more solvent accessible. In K4 dimethylation, the addition of the second methyl group restores fuzzy contacts with DNA and slightly reduces contact with water. The addition of the third methyl group has little impact on $\mathrm{R} 8$ contacts, i.e., $\mathrm{K}^{4} \mathrm{Me} 2$ and $\mathrm{K}^{4} \mathrm{Me} 3$ have a similar effect on R8.

Next, we evaluated $R 8$ contacts in $\mathrm{K}^{9,27}$ Me3 and $\mathrm{S}^{10,28} \mathrm{P}$. In both systems, these modifications are in Segments I and II. We expected that a PTM in the vicinity of R8 would have a significant impact on its interactions. The $\mathrm{K}^{9,27}$ Me3 affects $\mathrm{R} 8$ in almost the same way as $\mathrm{K}^{4} \mathrm{Me1}$, detaching the R8 from the DNA and making it solvent accessible. However, the phosphorylation at $S 10$ in $S^{10,28}$ pinds $R 8$ to the DNA and maintains contact with water. Through contact analysis, we discovered that the nature of the modificationtrimethylation versus phosphorylation-impacts the $\mathrm{R} 8$ contacts more than the proximity of the modification.

Finally, we studied the $\mathrm{K}^{4,36}{ }_{\mathrm{Me} 3} \mathrm{~K}^{9,27}{ }_{\mathrm{Me} 1}$ system which has modifications in all segments. We discovered that methylations of four residues spaced along the entire length of the $\mathrm{H} 3$ tail anchored the tail, allowing $\mathrm{R} 8$ to have persistent contact with a single DNA residue (d45). All interactions with water and ions were lost.

Using R8 contact analysis, we demonstrated that a single modification of a histone tail residue can alter the tail's contact at multiple locations. The segmental and yet flexible nature of the H3 tail supports the fuzzy contacts with DNA and the solvent. More importantly, this simple contact analysis of an $\mathrm{H} 3$ tail residue proves the effect of PTMs on regulating the chromatin structure. 


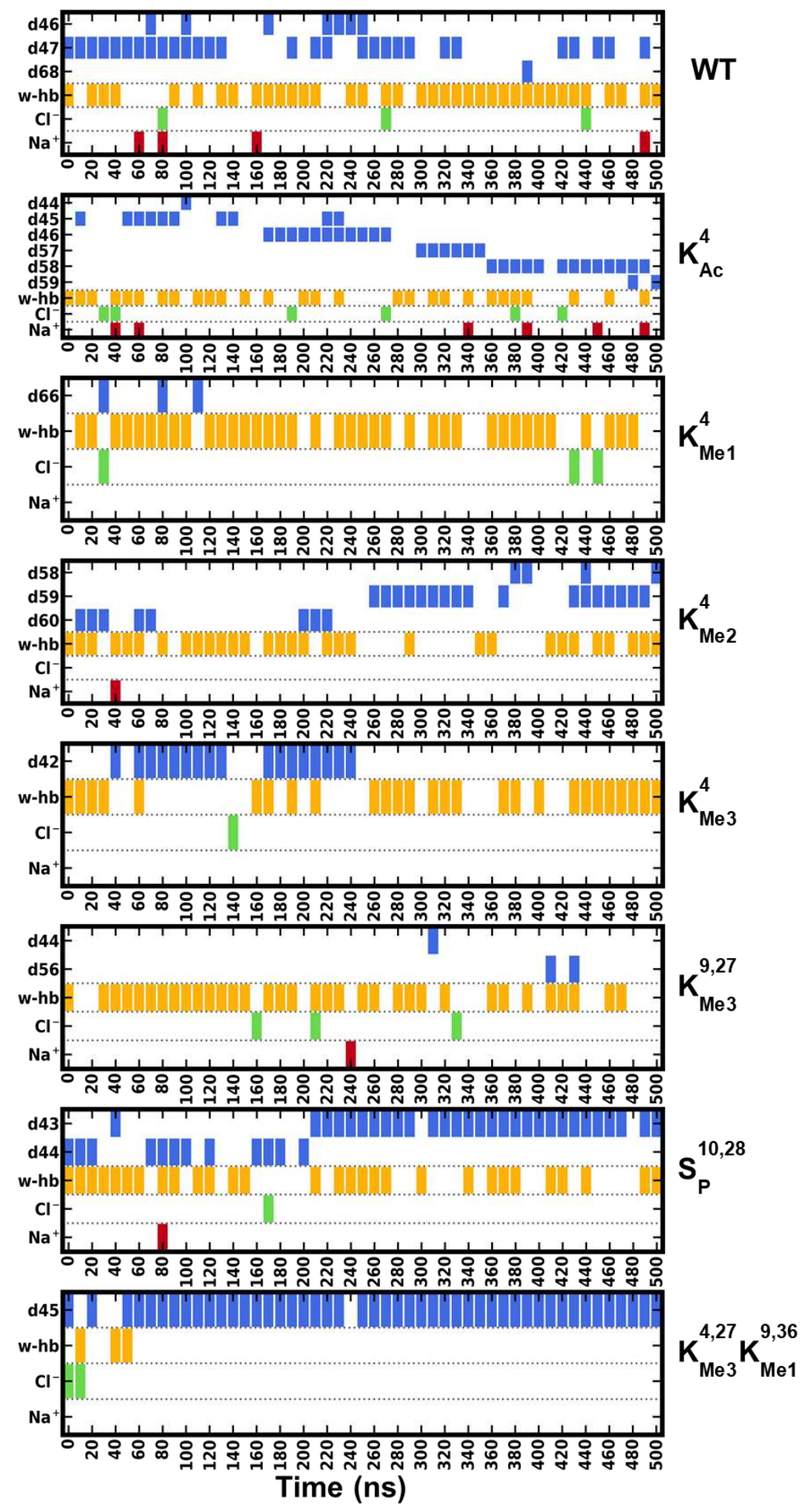

Figure 5. Time propogation of SHL1 H3 R8 contacts over the last 500 ns (20 ns increments) of the simulation, with DNA residues ( $\mathrm{d}^{* *}$, blue), h-bonded water (w-hb, yellow), anion $\left(\mathrm{Cl}^{-}\right.$, green), and cation $\left(\mathrm{Na}^{+}, \mathrm{red}\right)$ for all eight systems. 
To quantify the binding strength of the Segment I residues to DNA, we used steered MD simulations to compute the change in free energy $(\Delta G)$ of their unbound and bound states. In each system, we applied a force to the COM of Segment I residues to pull the tail away from the DNA until the tail was fully extended off the nucleosome. The resulting free energy profiles of the eight systems are shown in Figure 6.

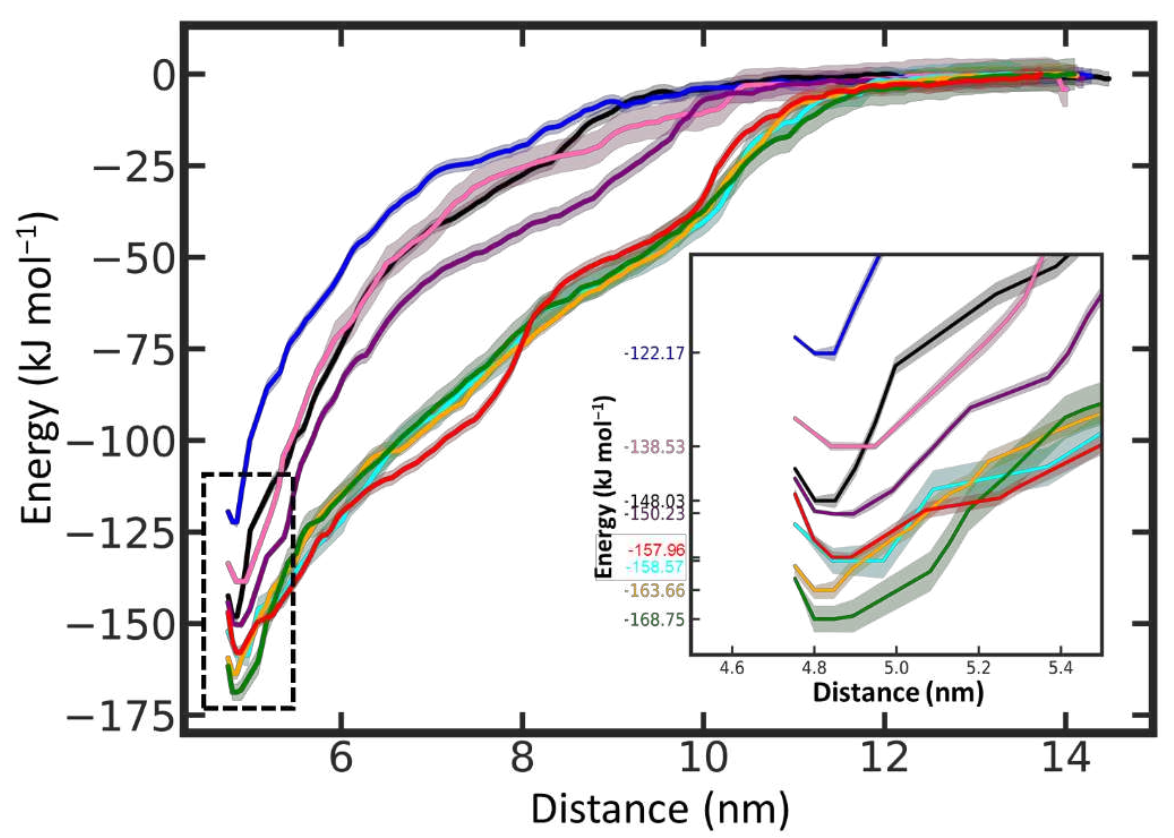

Figure 6. Potential of mean force (PMF) curves for the WT and seven PTM systems. The change in the free energy $(\Delta G)$ as a function of distance or the reaction coordinate in the unbinding of $\mathrm{H} 3$ tail's Segment I with DNA. The inset shows a zoomed-in view of the curves within 4-6 nm of the reaction coordinate. Solid lines indicate the PMF curve, and shadows indicate the deviation in the $\Delta G$ along the reaction coordinate. Color scheme: WT (black), $\mathrm{K}^{4}{ }_{\mathrm{Ac}}$ (pink), $\mathrm{K}^{4}{ }_{\mathrm{Me} 1}$ (purple), $\mathrm{K}^{4}{ }_{\mathrm{Me}}$ (cyan), $\mathrm{K}^{4}{ }_{\mathrm{Me}}$ (orange), $\mathrm{K}^{9,27}{ }_{\mathrm{Me}}$ (red), $\mathrm{S}^{10,28_{\mathrm{P}}}$ (blue), and $\mathrm{K}^{4,27}{ }_{\text {Me3 }} \mathrm{K}^{9,36}{ }_{\mathrm{Me} 1}$ (green).

The free energy curves were varied from contact distance $(4 \AA)$ to noninteracting distances $(>12 \mathrm{~nm})$ along the reaction coordinate, which is defined by the distance between the DNA and the H3 Segment I. The minimum energy in the curve corresponds to the free energy of binding between DNA and the $\mathrm{H} 3$ tail; the lower the change in free energy, the higher the binding energy.

The weakest binding was observed for the $\mathrm{S}^{10,28} \mathrm{p} \mathrm{H} 3$ tail, which is $+25.9 \mathrm{kJmol}^{-1}$ (less stable) compared to the WT Segment I. The only other system with binding strength weaker than WT was $\mathrm{K}^{4} \mathrm{Ac}$, which occurs because lysine's positive charge is neutralized by the addition of the acetyl group, thus eliminating its electrostatic interaction with oppositely charged DNA. Conversely, all three K4 methylation states increased binding compared to the WT because methylations do not affect the net positive charge on the residue but increase hydrophobicity and size.

The higher binding energies observed in super methylated $\mathrm{K}^{9,27}{ }_{\mathrm{Me}}$ and $\mathrm{K}^{4,27}{ }_{\mathrm{Me}} \mathrm{K}^{9,36}{ }_{\mathrm{Me} 1}$ systems show that multiple methylated residues stabilize the DNA-H3 tail binding. Additional evidence for this observation comes from summing over residue contacts of the DNA-H3 tails for all eight systems; the $\mathrm{K}^{4,27}{ }_{\mathrm{Me}} \mathrm{K}^{9,36}{ }_{\mathrm{Me} 1}$ makes the highest total contact with DNA. The PMF results confirm that modifications of residues in all three $\mathrm{H} 3$ tail segments affect the tail's interactions with DNA. 


\subsection{Segment II forms bands of robust contacts}

The restricted flexibility of Segment II is attributed to the slightly shorter 14 peptide length in the H3 tail's midsection, hinged by P16 and P30 on either end. Only two regions in Segment II make strong contacts with the DNA. First, sidechains of residues A21 and T22 point toward the DNA, while the tail conformation causes adjacent K23 to point away despite its positive charge (Figure 7). This observation demonstrates that not all positively charged residues in the $\mathrm{H} 3$ histone tail default to interacting with DNA and that amino acid side chain configuration is critical to the histone tail's behavior. The conformation of this region remains the same in all eight systems. Second, residue pair R26-K27 are oriented such that the R26 side chain interacts strongly with the DNA while K27 is pointed away from DNA, allowing it to be methylated. The orientation of these two residues remains unchanged in $\mathrm{K}^{9,27}$ Me3 and $\mathrm{K}^{4,36}{ }_{\mathrm{Me} 3} \mathrm{~K}^{9,27}$ Me1 even when $\mathrm{K} 27$ is methylated. Moreover, fixed conformations in A2-K23 and R26-K27 regions prevent the H3 tail from entirely collapsing and flattening onto the DNA. The lack of conformational freedom is evident in all eight systems.

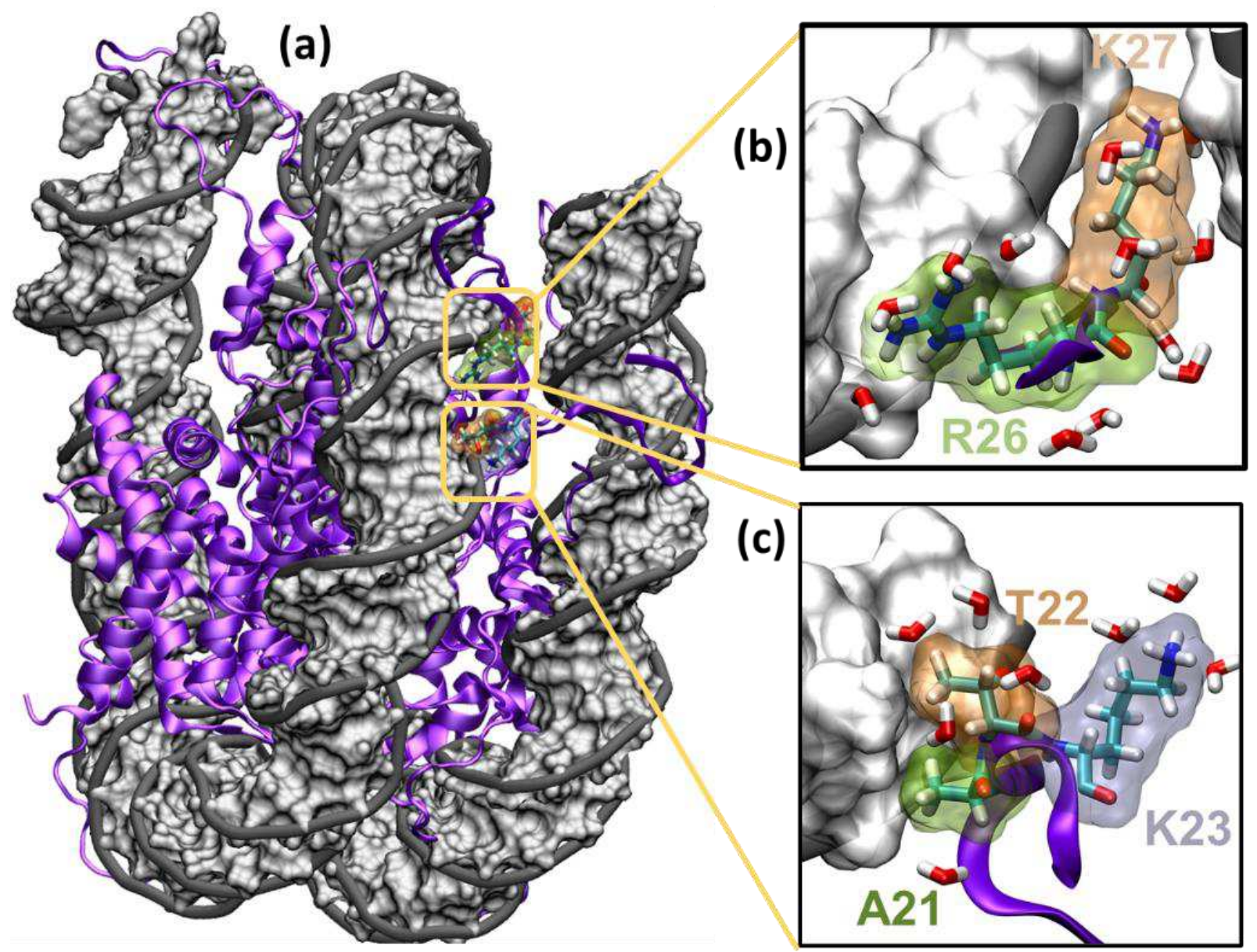

Figure 7. Conformation of $\mathrm{H} 3$ tail A21-T22-K23 residue triplet and R26-K27 residue pair. WT system shown. (a) whole nucleosome with histone proteins colored in purple. (b) zoomed in view of R26-K27 residue pair. (c) zoomed in view of the A21-T22-K23 residue triplet. In zoomed in views, neighboring waters are shown.

\subsection{Post translational modifications effect $\mathrm{H} 3$ dynamics}

Our results showed that methylation, acetylation, and phosphorylation of $\mathrm{H} 3$ tail residue(s) change the tail's contacts and binding strength with DNA. Next, we evaluated the how the PTMs alter the tail's dynamics 
(Figure 8). A comparison of the RMSF values of the $\mathrm{H} 3$ tail residues shows that each modification has a unique effect on tail dynamics (Figure 8a).

We begin our discussion of the $\mathrm{H} 3$ tail dynamics by comparing the $\mathrm{C}_{\alpha}$ RMSF values of the WT H3 tail with the methylated systems. We noticed that monomethylation of $\mathrm{K} 4$ residue resulted in the largest fluctuations in the H3 tail with RMSF values in the 4-13 $\AA$ range compared to WT (4-6 ̊) and other methylated tails. To determine the cause of these fluctuations, we generated time-lapsed snapshots in $25 \mathrm{~ns}$ intervals of the tail's motion over the last 500 ns of the trajectory. We observed that the tail does not detach from the DNA to adopt new conformations. Instead, the tail undergoes a "swing motion" while maintaining its collapsed conformation (Figures 8b-8c). The amplitude of the tail's swing from 5-10 $\AA$ over a 10-20 ns timescale agrees with the timescales reported for medium-range loop motions observed in the active site conformational change in enzymes, hinge motions, and loop formation. Moreover, the swinging motion occurs in all three segments, indicating that a single K4 monomethylation can alter the dynamics of the entire H3 tail. The tail's segmental divide at the prolines is evident from the RMSF data; P16 has the lowest fluctuation of the tail.

Additional $\mathrm{K} 4$ methylations in $\mathrm{K}^{4} \mathrm{Me} 2$ and $\mathrm{K}^{4} \mathrm{Me}$ show progressively smaller fluctuations, but the fluctuations are still the largest among Segment I residues (Figure 8a). If the number of methylated residues is increased to two in $\mathrm{K}^{9,27} \mathrm{Me} 3$ or multiple in $\mathrm{K}^{4,27} \mathrm{Me} \mathrm{K}^{9,36} \mathrm{Me} 1$, we observed that tail fluctuations were diminished (Figure $8 \mathrm{f}-8 \mathrm{~g}$ ). A comparison of the time-lapsed snapshots of the methylated systems shows that the fluctuations are segmental and hinged at the prolines in all cases. Since these peptides are tethered to the histone core particle and interact with the locally wrapped DNA, it is striking that any differences can be observed between the systems with the net charge-neutral addition of a single methyl group. Collectively, our results from $\mathrm{H} 3$ methylations suggest two key points: (ii) single-residue methylation can alter the dynamics of the entire chain, and (ii) multiple methylated residues reduce the tail's fluctuations.

Unlike $\mathrm{H}_{3} \mathrm{~K}^{4} \mathrm{Me}$, the fluctuations in $\mathrm{H}_{3} \mathrm{~K}^{4}{ }_{\mathrm{Ac}}$ tail residues are much smaller, evident from 2-6 $\AA \mathrm{C}_{\alpha} \mathrm{RMSF}$ values. The explanation for this difference lies in the chemical nature of the modification. In acetylation, the loss of positive charge in the lysine side chain results in the loss of DNA contacts (Figures 4 and 5) and lower binding energy (Figure 6). Thus, the loss of positive charge at the K4 side chain appears to affect the strength of DNA-residue binding, but not the tail dynamics (Figure 8h).

The negative charges at residues 10 and 28 in $S^{10,28} \mathrm{P}$ did not affect the entire tail's dynamics. Segment I fluctuations remained almost unchanged and were similar to WT, while there were higher local fluctuations in the RMSF values (4-6 A) for residues 29-33. The local fluctuation included the P30 residue, which remained in unchanged like most other modifications. As in previous fluctuations, the local increase in RMSF values is due to the swinging motion around residues 29-33 in an otherwise collapsed tail (Figure 8i). 


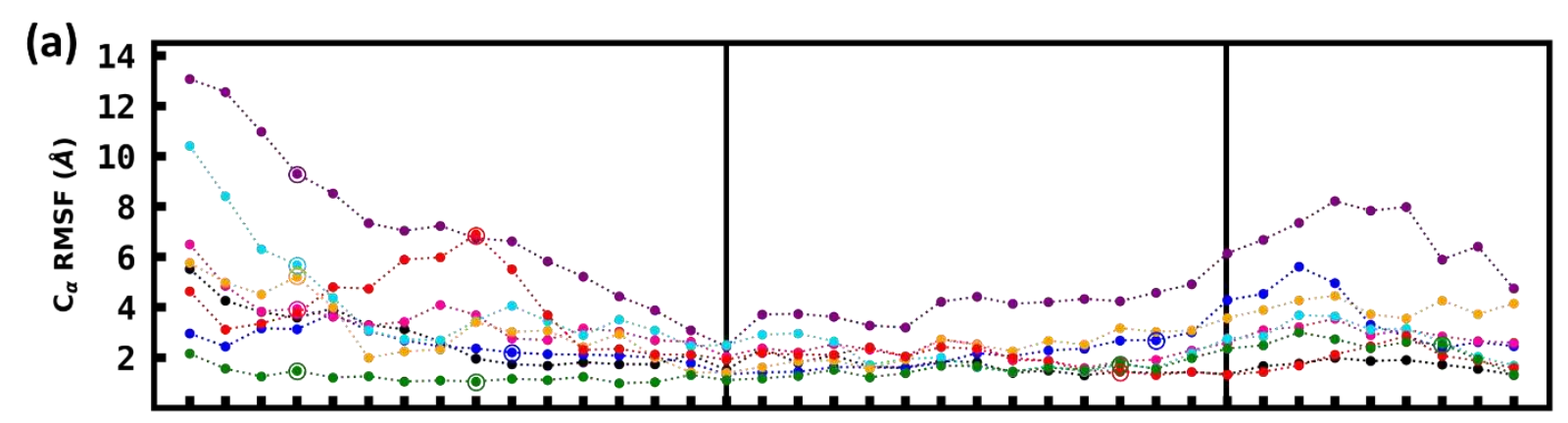

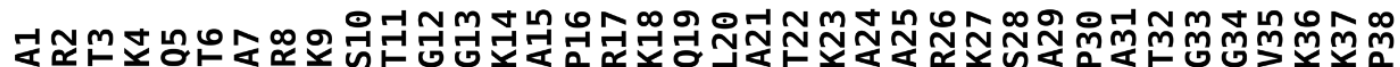
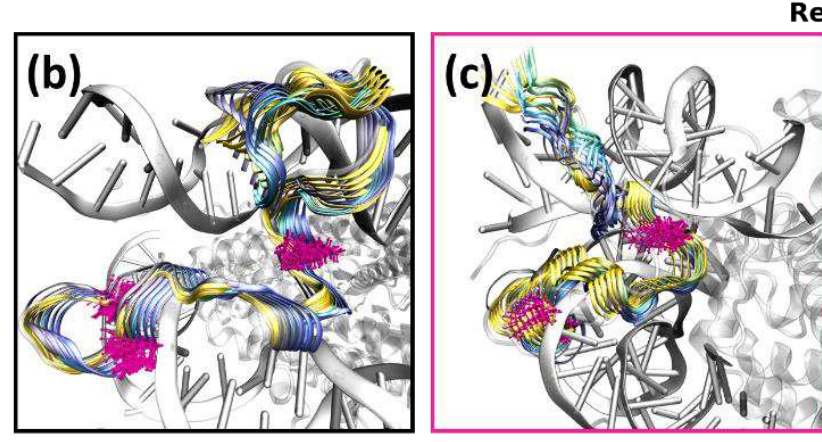

Residue
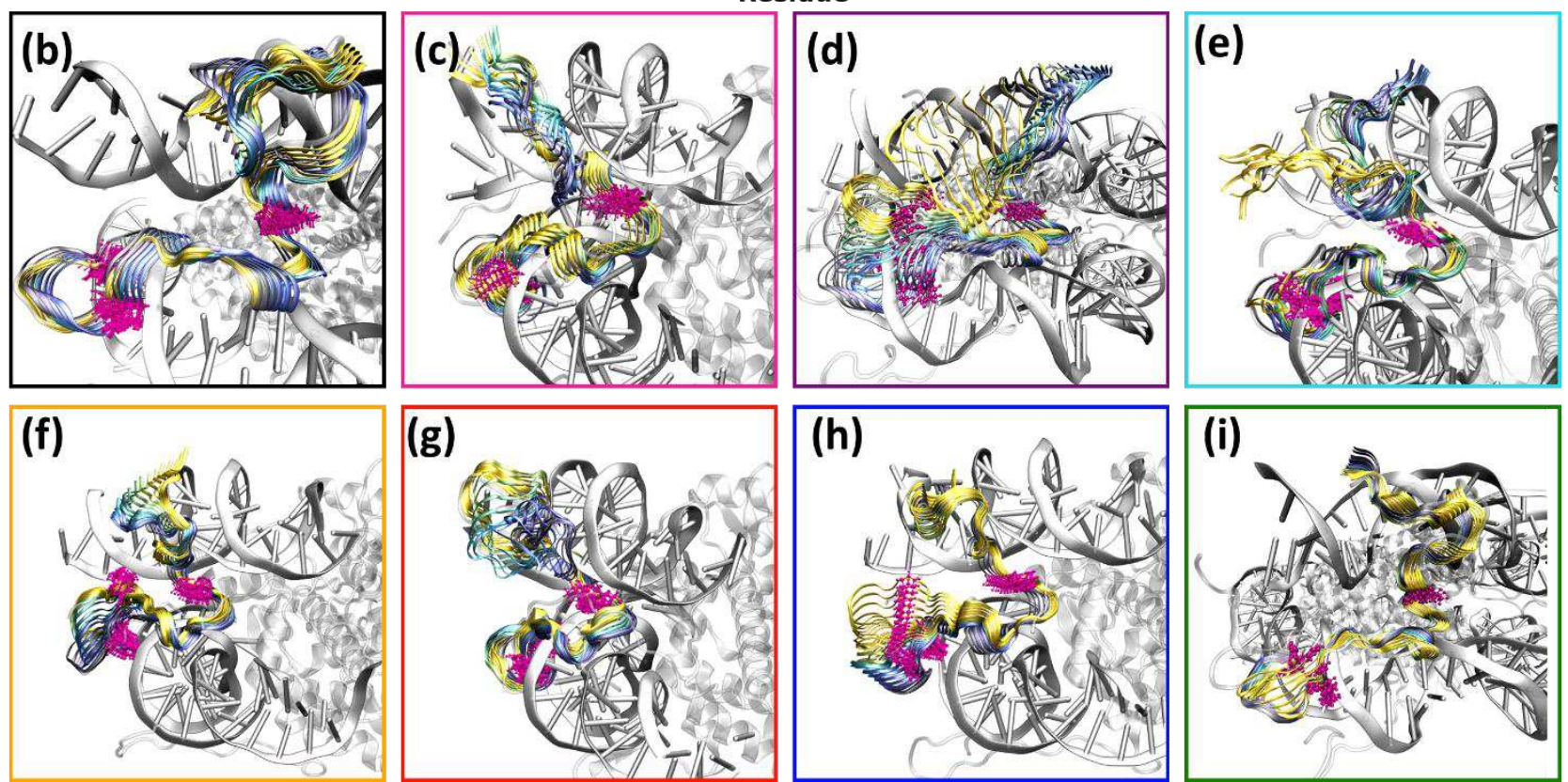

Figure 8. Dynamics of the $\mathrm{H} 3$ tail. (a) RMSF of $\mathrm{C}_{\alpha}$ atoms of the tail residues in all eight systems over the last $500 \mathrm{~ns}$ of the simulation. Double circles are shown at the location of a PTM. Time-elapsed snapshots of H3 tail dynamics for (b) WT (black), (c) $\mathrm{K}^{4} \mathrm{Me} 1$ (purple), (d) $\mathrm{K}^{4} \mathrm{Me2}$ (cyan), (e) $\mathrm{K}^{4}{ }_{\mathrm{Me} 3}$ (orange), (f) $\mathrm{K}^{9,27} \mathrm{Me3}$ (red) (g) $\mathrm{K}^{4,27} \mathrm{Me} \mathrm{K}^{9,36}{ }_{\mathrm{Me}}$ (green) (h) $\mathrm{K}^{4} \mathrm{Ac}_{\mathrm{c}}$ (pink) and (i) $\mathrm{S}^{10,28} \mathrm{P}$ (blue). The snapshots are shown for the last $500 \mathrm{~ns}$ of the trajectory at $25 \mathrm{~ns}$ intervals (purple to yellow, ribbon), DNA (gray, ribbon), histones (gray, ribbon), and H3 tail proline residues (balls, pink). 


\section{CONCLUSIONS}

This study provides evidence that the $\mathrm{N}$-terminal $\mathrm{H} 3$ tail acquires a collapsed conformation at SHL1, and despite its intrinsically disordered structure, the tail shows a tripartite segmental behavior. The $\mathrm{H} 3$ tail can be divided into Segments I, II, and III, with proline residues P16, P30, and P38 identified as segmental dividers. Each segment has a different length and composition of charged residues available for PTMs. The flexibility of the $\mathrm{H} 3$ tail segments decreases from the tip to the core. Segment I is most dynamic and shows contact variations with residue modification. Segment II is less flexible, evident from the well-defined DNA contacts in the WT that remain essentially unchanged by PTMs. The eight amino acid Segment III is the shortest section of $\mathrm{H} 3$, contains the least number of charged residues, and is the most restricted. We observed that methylation of a single tail residue could alter the tail dynamics while multiple methylated residues reduce the tail's fluctuations; however, the tripartite segmental behavior of the $\mathrm{H} 3$ tail is largely preserved. Although the exact mechanisms of addition and removal of PTMs on the histone tails are still elusive, our work provides evidence that different multiple PTM combinations alter the dynamics of the $\mathrm{H} 3$ tails.

\section{ACKNOWLEDGEMENTS}

This work is supported by grants: National Science Foundation (NSF) CAREER CBET-1453312, NIH R01GM134102, and NSF DMR-1757749 (REU) to S.N.; NSF GRFP 05316 to K.M.P.; and NIH grant R01CA140522 to M.S.C. Computational resources were provided by the Information and Technology Services at Syracuse University (Eric Sedore, Larne Pekowsky, and Michael R Brady), and the Extreme Science and Engineering Discovery Environment (XSEDE), which is supported by National Science Foundation grant number ACl-1053575.

Supporting Information contains: details of the MD systems; the force field parameters of lysine acetylation, monomethylation, dimethylation, and trimethylation; snapshots of collapsed SHL1 H3 tail; Ramachandran plots of proline residues in WT and PTM systems; RMSF of H3m systems; DNA-H3 tail contact map for H3m systems; Ramachandran plots for WT and PTM systems; end-to-end distances and radius of gyration plots for WT and PTM systems.

\section{REFERENCES}

1. Luger, K.; Rechsteiner, T. J.; Richmond, T. J., Expression and Purification of Recombinant Histones and Nucleosome Reconstitution. In Chromatin Protocols, Becker, P. B., Ed. Humana Press: Totowa, NJ, 1999; pp 1-16.

2. Richmond, T. J.; Davey, C. A., The structure of DNA in the nucleosome core. Nature 2003, 423 (6936), 145-150.

3. Marino-Ramirez, L.; Kann, M. G.; Shoemaker, B. A.; Landsman, D., Histone structure and nucleosome stability. Expert Review Of Proteomics 2005, 2 (5), 719-729.

4. Andrews, A. J.; Luger, K., Nucleosome Structure(s) and Stability: Variations on a Theme. In Annual Review Of Biophysics, VOL 40, Rees, D. C.; Dill, K. A.; Williamson, J. R., Eds. 2011; Vol. 40, pp 99117.

5. Iwasaki, W.; Miya, Y.; Horikoshi, N.; Osakabe, A.; Taguchi, H.; Tachiwana, H.; Shibata, T.; Kagawa, W.; Kurumizaka, $\mathrm{H}$., Contribution of histone $\mathrm{N}$-terminal tails to the structure and stability of nucleosomes. FEBS Open Bio 2013, 3, 363-369.

6. Cutter, A. R.; Hayes, J. J., A brief review of nucleosome structure. FEBS Lett 2015, 589 (20 Pt A), 2914-22.

7. Luger, K.; Mäder, A. W.; Richmond, R. K.; Sargent, D. F.; Richmond, T. J., Crystal structure of the nucleosome core particle at $2.8 \AA$ resolution. Nature 1997, 389 (6648), 251-260.

8. Zhou, K. D.; Gaullier, G.; Luger, K., Nucleosome structure and dynamics are coming of age. Nature Structural and Molecular Biology 2019, 26 (1), 3-13. 
9. Davey, C. A.; Sargent, D. F.; Luger, K.; Maeder, A. W.; Richmond, T. J., Solvent Mediated Interactions in the Structure of the Nucleosome Core Particle at 1.9A Resolution. Journal of Molecular Biology 2002, 319 (5), 1097-1113.

10. Bai, X. C.; McMullan, G.; Scheres, S. H. W., How cryo-EM is revolutionizing structural biology. Trends in Biochemical Sciences 2015, 40 (1), 49-57.

11. Chua, E. Y. D.; Vogirala, V. K.; Inian, O.; Wong, A. S. W.; Nordenskiold, L.; Plitzko, J. M.; Danev, R.; Sandin, S., 3.9 angstrom structure of the nucleosome core particle determined by phase-plate cryoEM. Nucleic Acids Research 2016, 44 (17), 8013-8019.

12. Wilson, M. D.; Costa, A., Cryo-electron microscopy of chromatin biology. Acta Crystallographica Section D-Structural Biology 2017, 73, 541-548.

13. Gao, M.; Nadaud, P. S.; Bernier, M. W.; North, J. A.; Hammel, P. C.; Poirier, M. G.; Jaroniec, C. P., Histone H3 and $\mathrm{H} 4 \mathrm{~N}$-Terminal Tails in Nucleosome Arrays at Cellular Concentrations Probed by Magic Angle Spinning NMR Spectroscopy. Journal of the American Chemical Society 2013, 135 (41), 15278-15281.

14. Wei, S.; Falk, S. J.; Black, B. E.; Lee, T.-H., A novel hybrid single molecule approach reveals spontaneous DNA motion in the nucleosome. Nucleic Acids Research 2015, 43 (17), e111-e111.

15. Shi, X.; Prasanna, C.; Nagashima, T.; Yamazaki, T.; Pervushin, K.; Nordenskiold, L., Structure and Dynamics in the Nucleosome Revealed by Solid-State NMR. Angew Chem Int Ed Engl 2018, 57 (31), 9734-9738.

16. Wang, X. Y.; Moore, S. C.; Laszckzak, M.; Ausio, J., Acetylation increases the alpha-helical content of the histone tails of the nucleosome. Journal Of Biological Chemistry 2000, 275 (45), 35013-35020.

17. Feng, Y.; Wang, J. X.; Asher, S.; Hoang, L.; Guardiani, C.; Ivanov, I.; Zheng, Y. G., Histone H4 Acetylation Differentially Modulates Arginine Methylation by an in Cis Mechanism. Journal Of Biological Chemistry 2011, 286 (23), 20323-20334.

18. Potoyan, D. A.; Papoian, G. A., Energy Landscape Analyses of Disordered Histone Tails Reveal Special Organization of Their Conformational Dynamics. Journal of the American Chemical Society 2011, 133 (19), 7405-7415.

19. Kampf, K.; Izmailov, S. A.; Rabdano, S. O.; Groves, A. T.; Podkorytov, I. S.; Skrynnikov, N. R., What Drives N-15 Spin Relaxation in Disordered Proteins? Combined NMR/MD Study of the H4 Histone Tail. Biophysical Journal 2018, 115 (12), 2348-2367.

20. Huang, H.; Lin, S.; Garcia, B. A.; Zhao, Y. M., Quantitative Proteomic Analysis of Histone Modifications. Chemical Reviews 2015, 115 (6), 2376-2418.

21. Baxevanis, A. D.; Landsman, D., Histone sequence database: A compilation of highly-conserved nucleoprotein sequences. Nucleic Acids Research 1996, 24 (1), 245-247.

22. Altschul, S. F.; Madden, T. L.; Schaffer, A. A.; Zhang, J. H.; Zhang, Z.; Miller, W.; Lipman, D. J., Gapped BLAST and PSI-BLAST: a new generation of protein database search programs. Nucleic Acids Research 1997, 25 (17), 3389-3402.

23. Walker, I. O., Differential dissociation of histone tails from core chromatin. (0006-2960 (Print)).

24. Shaytan, A. K.; Armeev, G. A.; Goncearenco, A.; Zhurkin, V. B.; Landsman, D.; Panchenko, A. R., Coupling between Histone Conformations and DNA Geometry in Nucleosomes on a Microsecond Timescale: Atomistic Insights into Nucleosome Functions. J Mol Biol 2016, 428 (1), 221-237.

25. Rabdano, S. O.; Shannon, M. D.; Izmailov, S. A.; Gonzalez Salguero, N.; Zandian, M.; Purusottam, R. N.; Poirier, M. G.; Skrynnikov, N. R.; Jaroniec, C. P., Histone H4 Tails in Nucleosomes: a Fuzzy Interaction with DNA. Angewandte Chemie - International Edition 2021, 60 (12), 6480-6487.

26. Furukawa, A.; Wakamori, M.; Arimura, Y.; Ohtomo, H.; Tsunaka, Y.; Kurumizaka, H.; Umehara, T.; Nishimura, Y., Acetylated histone $\mathrm{H} 4$ tail enhances histone $\mathrm{H} 3$ tail acetylation by altering their mutual dynamics in the nucleosome. Proceedings Of The National Academy Of Sciences Of The United States Of America 2020, 117 (33), 19661-19663.

27. Martin, C.; Zhang, Y., The diverse functions of histone lysine methylation. Nature Reviews Molecular Cell Biology 2005, 6 (11), 838-849.

28. Nowak, S. J.; Corces, V. G., Phosphorylation of histone H3: a balancing act between chromosome condensation and transcriptional activation. Trends In Genetics 2004, 20 (4), 214-220.

29. Cosgrove, M. S.; Boeke, J. D.; Wolberger, C., Regulated nucleosome mobility and the histone code. Nature Structural \& Molecular Biology 2004, 11 (11), 1037-1043.

30. Munshi, A.; Shafi, G.; Aliya, N.; Jyothy, A., Histone modifications dictate specific biological readouts. Journal Of Genetics And Genomics 2009, 36 (2), 75-88. 
31. Bowman, G. D.; Poirier, M. G., Post-Translational Modifications of Histones That Influence Nucleosome Dynamics. Chemical Reviews 2015, 115 (6), 2274-2295.

32. Lawrence, M.; Daujat, S.; Schneider, R., Lateral Thinking: How Histone Modifications Regulate Gene Expression. Trends In Genetics 2016, 32 (1), 42-56.

33. Nadal, S.; Raj, R.; Mohammed, S.; Davis, B. G., Synthetic post-translational modification of histones. Current Opinion In Chemical Biology 2018, 45, 35-47.

34. Rice, J. C.; Allis, C. D., Histone methylation versus histone acetylation: new insights into epigenetic regulation. Current Opinion In Cell Biology 2001, 13 (3), 263-273.

35. Collepardo-Guevara, R.; Portella, G.; Vendruscolo, M.; Frenkel, D.; Schlick, T.; Orozco, M., Chromatin Unfolding by Epigenetic Modifications Explained by dramatic impairment of internucleosome interactions: A multiscale computational study. Journal of the American Chemical Society 2015, 137 (32), 10205-10215.

36. Stutzer, A.; Liokatis, S.; Kiesel, A.; Schwarzer, D.; Sprangers, R.; Soding, J.; Selenko, P.; Fischle, W., Modulations of DNA Contacts by Linker Histones and Post-translational Modifications Determine the Mobility and Modifiability of Nucleosomal H3 Tails. Mol Cell 2016, 61 (2), 247-59.

37. Morrison, E. A.; Bowerman, S.; Sylvers, K. L.; Wereszczynski, J.; Musselman, C. A., The conformation of the histone $\mathrm{H} 3$ tail inhibits association of the BPTF PHD finger with the nucleosome. Elife 2018, 7.

38. Zhou, B. R.; Feng, H.; Ghirlando, R.; Kato, H.; Gruschus, J.; Bai, Y., Histone H4 K16Q mutation, an acetylation mimic, causes structural disorder of its $\mathrm{N}$-terminal basic patch in the nucleosome. $\mathrm{J} \mathrm{Mol}$ Biol 2012, 421 (1), 30-7.

39. Scott, W. A.; Campos, E. I., Interactions with histone H3 \& tools to study them. Frontiers in Cell and Developmental Biology 2020, 8, 701.

40. Biswas, M.; Langowski, J.; Bishop, T. C., Atomistic simulations of nucleosomes. Wiley Interdisciplinary Reviews: Computational Molecular Science 2013, 3 (4), 378-392.

41. Li, Z.; Kono, H., Distinct Roles of Histone H3 and H2A Tails in Nucleosome Stability. Sci Rep 2016, $6,31437$.

42. Stützer, A.; Liokatis, S.; Kiesel, A.; Schwarzer, D.; Sprangers, R.; Söding, J.; Selenko, P.; Fischle, W., Modulations of DNA Contacts by Linker Histones and Post-translational Modifications Determine the Mobility and Modifiability of Nucleosomal H3 Tails. Molecular Cell 2016, 61 (2), 247-259.

43. Ikebe, J.; Sakuraba, S.; Kono, H., H3 Histone Tail Conformation within the Nucleosome and the Impact of K14 Acetylation Studied Using Enhanced Sampling Simulation. PLoS Comput Biol 2016, 12 (3), e1004788.

44. Böhm, L.; Crane-Robinson, C., Proteases as structural probes for chromatin: The domain structure of histones: Review. Bioscience Reports 1984, 4 (5), 365-386.

45. Rosenberg, N. L.; Smith, R. M.; Rill, R. L., The action of chymotrypsin on nucleosome cores histone products and conformational effects of limited digestion. The Journal of biological chemistry 1986, 261 (26), 2375-2383.

46. Luger, K.; Richmond, T. J., The histone tails of the nucleosome. Current Opinion in Genetics \& Development 1998, 8 (2), 140-146.

47. Wang, Z. A.; Cole, P. A., The Chemical Biology of Reversible Lysine Post-translational Modifications. Cell Chem Biol 2020, 27 (8), 953-969.

48. Luo, M., Chemical and Biochemical Perspectives of Protein Lysine Methylation. Chem Rev 2018, $118(14), 6656-6705$.

49. Cosgrove, M. S., Histone proteomics and the epigenetic regulation of nucleosome mobility. Expert Review of Proteomics 2007, 4 (4), 465-478.

50. Cosgrove, M. S.; Wolberger, C., How does the histone code work? Biochemistry and Cell Biology 2005, 83 (4), 468-476.

51. Liu, H.; Duan, Y., Effects of posttranslational modifications on the structure and dynamics of histone H3 N-terminal Peptide. Biophysical Journal 2008, 94 (12), 4579-85.

52. Young, N. L.; DiMaggio, P. A.; Plazas-Mayorca, M. D.; Baliban, R. C.; Floudas, C. A.; Garcia, B. A., High throughput characterization of combinatorial histone codes. Mol Cell Proteomics 2009, 8 (10), 2266-84.

53. Ozturk, M. A.; De, M.; Cojocaru, V.; Wade, R. C., Chromatosome Structure and Dynamics from Molecular Simulations. In Annual Review Of Physical Chemistry, VOL 71, Johnson, M. A.; Martinez, T. J., Eds. 2020; Vol. 71, pp 101-119. 
54. Clark, R. S. B.; Bayir, H.; Jenkins, L. W., Posttranslational protein modifications. Critical Care Medicine 2005, 33 (12), S407-S409.

55. Erler, J.; Zhang, R.; Petridis, L.; Cheng, X.; Smith, J. C.; Langowski, J., The role of histone tails in the nucleosome: a computational study. Biophysical journal 2014, 107 (12), 2911-2922.

56. Abraham, M. J.; Murtola, T.; Schulz, R.; Páll, S.; Smith, J. C.; Hess, B.; Lindahl, E., GROMACS: High performance molecular simulations through multi-level parallelism from laptops to supercomputers. SoftwareX 2015, 1-2, 19-25.

57. Huang, J.; MacKerell, A. D., Jr., CHARMM36 all-atom additive protein force field: validation based on comparison to NMR data. (1096-987X (Electronic)).

58. Jorgensen, W. L.; Chandrasekhar, J.; Madura, J. D.; Impey, R. W.; Klein, M. L., Comparison of simple potential functions for simulating liquid water. The Journal of Chemical Physics 1983, 79 (2), 926-935.

59. Grauffel, C.; Stote, R. H.; Dejaegere, A., Force field parameters for the simulation of modified histone tails. J Comput Chem 2010, 31 (13), 2434-51.

60. Lee, J.; Cheng, X.; Swails, J. M.; Yeom, M. S.; Eastman, P. K.; Lemkul, J. A.; Wei, S.; Buckner, J.; Jeong, J. C.; Qi, Y.; Jo, S.; Pande, V. S.; Case, D. A.; Brooks, C. L., 3rd; MacKerell, A. D., Jr.; Klauda, J. B.; Im, W., CHARMM-GUI Input Generator for NAMD, GROMACS, AMBER, OpenMM, and CHARMM/OpenMM Simulations Using the CHARMM36 Additive Force Field. (1549-9626 (Electronic)).

61. Evans, D. J.; Holian, B. L., The nose-hoover thermostat. Journal of Chemical Physics 1985, 83 (8), 4069-4074.

62. Parrinello, M.; Rahman, A., Polymorphic transitions in single-crystals - a new molecular-dynamics method. Journal of Applied Physics 1981, 52 (12), 7182-7190.

63. Michaud-Agrawal, N.; Denning, E. J.; Woolf, T. B.; Beckstein, O., MDAnalysis: A toolkit for the analysis of molecular dynamics simulations. Journal of Computational Chemistry 2011, 32 (10), 2319-2327.

64. Michaud-Agrawal, N.; Denning, E. J.; Woolf, T. B.; Beckstein, O., Software News and Updates MDAnalysis: A Toolkit for the Analysis of Molecular Dynamics Simulations. Journal of Computational Chemistry 2011, 32 (10), 2319-2327.

65. Humphrey, W.; Dalke, A.; Schulten, K., VMD: Visual molecular dynamics. Journal Of Molecular Graphics \& Modelling 1996, 14 (1), 33-38.

66. Ghoneim, M.; Fuchs, H. A.; Musselman, C. A., Histone Tail Conformations: A fuzzy affair with DNA. Trends Biochem Sci 2021, 46 (7), 564-578. 


\section{Supplementary Files}

This is a list of supplementary files associated with this preprint. Click to download.

- Supportinglnfo10.pdf 